\title{
Targeting the environment
}

\author{
J. H. van Krieken ${ }^{1}$
}

(C) Springer-Verlag GmbH Germany 2017

The literature review in this issue deals with quite some papers that cover aspects of the tumor microenvironment in lymphoma [1]. This is actually a revival, because already many years ago, it became clear that the environment plays a crucial role in the maintenance and tumor progression of lymphomas. It was often stated that low grade lymphomas are probably more dependent on signals from other cells and the stroma than on their own neoplastic potential. The best example is still the low grade extranodal marginal zone lymphoma of the stomach that is dependent on T- lymphocytes, activated by the presence of Helicobacter pylori. The cure that follows eradication of the bacterium is therefore one of the greatest successes of therapy targeting the microenvironment. This could only happen thanks to the very precise observations of Peter Isaacson and the clever experiments he designed $[2,3]$.

The recent surge in articles on the microenvironment has at least partially been triggered by the success of new forms of immunotherapy and thus the focus lies on the role of lymphocytes, especially $\mathrm{T}$ - cells in tumors. This started in solid tumors like melanoma and colorectal cancer where results using new technologies to count lymphocytes could be found in high impact journal (articles from the 1970s doing the same with traditional methods were often not cited). Looking at the immunoarchitecture in lymphomas is a bit more complicated because the lymphoma cells themselves are already part of the immunoarchitecture and, importantly, likely still functional. Nevertheless, with the introduction of the new labeling and quantification techniques, it is now possible to gather more and new data that will certainly give us further insight. However, a complete picture must also take into account the antigen that is recognized by the antigen receptor of the tumor cells and the stromal components. By integrating all this information, we might find new approaches to treat especially low grade lymphomas. It takes a clever hematopathologist behind a microscope to do so, taking all the clinical and molecular knowledge into account.

\section{References}

1. van Krieken J (2017) New developments in the pathology of malignant lymphoma. A review of the literature published from May to August 2017. J Hematop: 10

2. Jaffe ES, Harris NL, Stein H, Isaacson PG (2008) Classification of lymphoid neoplasms: the microscope as a tool for disease discovery. Blood 112(12):4384-4399

3. Isaacson PG, Du MQ (2005) Gastrointestinal lymphoma: where morphology meets molecular biology. J Pathol 205:255-274

J. H. van Krieken

Han.vanKrieken@radboudumc.nl

1 Department of Pathology, Radboud University Medical Centre, P.O. Box 9101, 6500 HB Nijmegen, The Netherlands 